\title{
Widespread evidence of viral miRNAs targeting host pathways
}

\author{
Joseph W Carl Jr${ }^{1}$, Joanne Trgovcich², Sridhar Hannenhalli ${ }^{*}$ \\ From The Eleventh Asia Pacific Bioinformatics Conference (APBC 2013) \\ Vancouver, Canada. 21-24 January 2013
}

\begin{abstract}
Background: MicroRNAs (miRNA) are regulatory genes that target and repress other RNA molecules via sequencespecific binding. Several biological processes are regulated across many organisms by evolutionarily conserved miRNAs. Plants and invertebrates employ their miRNA in defense against viruses by targeting and degrading viral products. Viruses also encode miRNAs and there is evidence to suggest that virus-encoded miRNAs target specific host genes and pathways that may be beneficial for their infectivity and/or proliferation. However, it is not clear whether there are general patterns underlying cellular targets of viral miRNAs.

Results: Here we show that for several of the 135 known viral miRNAs in human viruses, the human genes targeted by the viral miRNA are enriched for specific host pathways whose targeting is likely beneficial to the virus. Given that viral miRNAs continue to be discovered as technologies evolve, we extended the investigation to 6809 putative miRNAs encoded by 23 human viruses. Our analysis further suggests that human viruses have evolved their miRNA repertoire to target specific human pathways, such as cell growth, axon guidance, and cell differentiation. Interestingly, many of the same pathways are also targeted in mice by miRNAs encoded by murine viruses. Furthermore, Human Cytomegalovirus (CMV) miRNAs that target specific human pathways exhibit increased conservation across CMV strains.
\end{abstract}

Conclusions: Overall, our results suggest that viruses may have evolved their miRNA repertoire to target specific host pathways as a means for their survival.

\section{Introduction}

MicroRNAs (miRNA) are $\sim 22$ nt non-coding regulatory genes that target other RNA molecules via sequence-specific hybridization, which results either in translation inhibition (an imperfect target miRNA sequence match) or in cleavage and degradation of the targeted RNA (a perfect target miRNA sequence match) [1]. Initially discovered in worms, miRNAs are now known to serve numerous critical regulatory functions in a wide spectrum of species including viruses [2], plants, and mammals [3]. In mammals, miRNAs play a regulatory role in processes as diverse as metabolism, immune response, cell death, proliferation, circadian rhythm, and hematopoiesis [4]

${ }^{1}$ Center for Bioinformatics and Computational Biology, University of

Maryland, College Park, MD, USA

Full list of author information is available at the end of the article
Figure 1 depicts all possible miRNA-mediated interactions between a virus and the host. A wide variety of species utilize their miRNAs to target and regulate endogenous pathways (Figure 1a) [5]. Plants, invertebrates [6], and vertebrates [7] utilize their miRNAs to target offending virus (Figure 1b). There are known instances of viruses using their endogenous miRNAs to target their own genes to evade host immune system's surveillance and maintain latency (Figure 1c) [8]. Finally, there are several known instances of viral miRNAs targeting host genes (Figure 1d). For instance, Epstein-Barr virus (EBV) encoded miR-BART5 targets the p53-regulated pro-apoptotic gene PUMA [9]. More recently, EBV miRNAs were determined to predominantly target cellular transcripts during latent infection; 531 sites were identified in 3492 cellular 3' UTRs
C Biomed Central

C 2013 Carl et al.; licensee BioMed Central Ltd. This is an open access article distributed under the terms of the Creative Commons Attribution License (http://creativecommons.org/licenses/by/2.0), which permits unrestricted use, distribution, and reproduction in any medium, provided the original work is properly cited. 


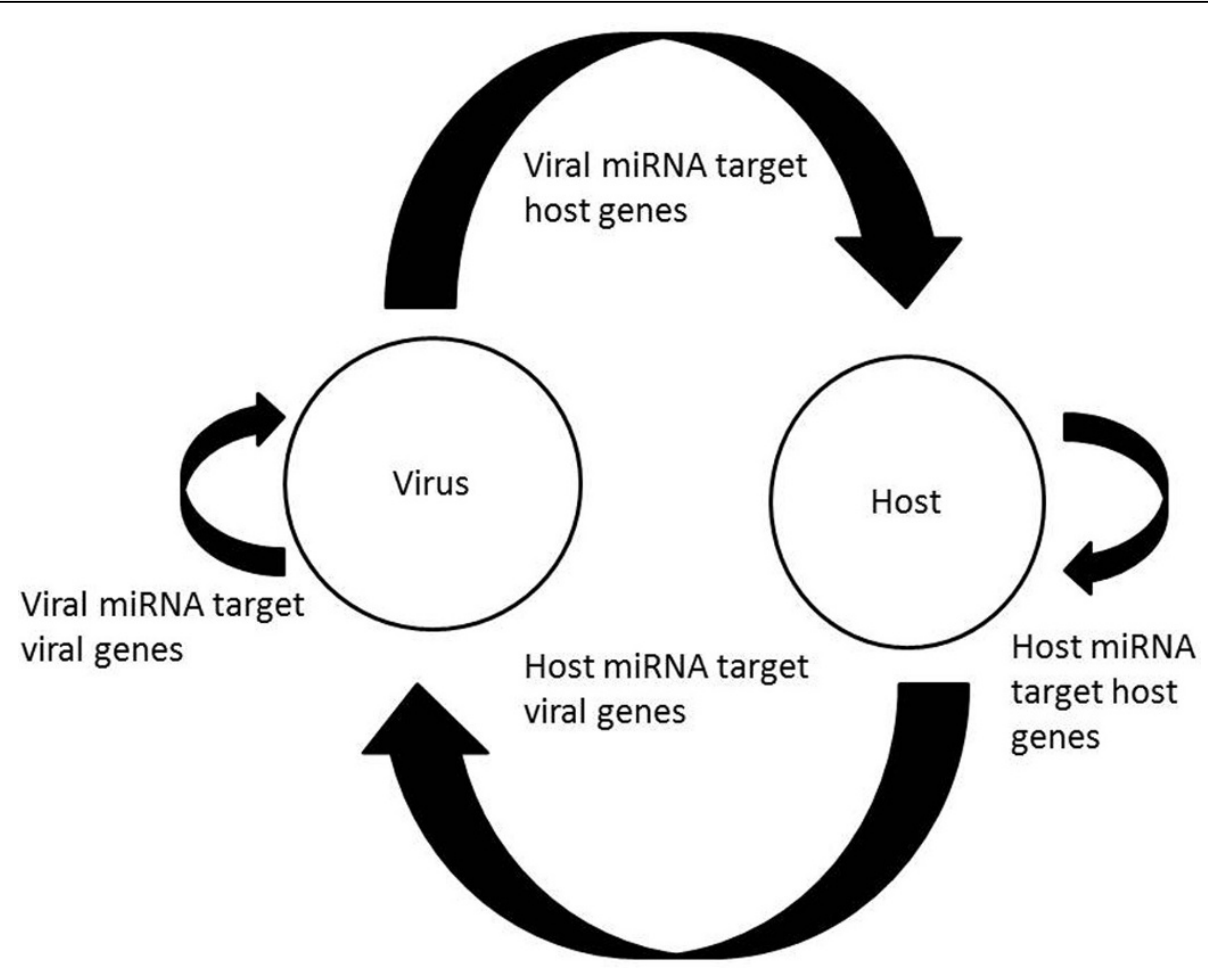

Figure 1 Possible interactions between miRNA and target genes. (a) Endogenous host miRNAs target specific host genes. (b) Host miRNAs target viral genes. (c) Virus encoded miRNAs target viral genes to mediate immune evasion or maintenance of latency. (d) Viral miRNAs have been shown to target specific host genes.

that contained seed matches to viral miRNAs; of them, 24 were experimentally confirmed [10]. More importantly, these target genes were enriched for cellular processes that facilitated the viral infection. This study suggested that the number of viral miRNA targets in the host may be much greater than previously assumed and are specific to host pathways.

Viruses depend upon the molecular machinery of the cells they infect. They have co-evolved with their host over millions of years and have had to adapt to the cellular environment, which in turn is evolving to evade viral infection. RNA viruses are limited in genomic size due in part to error prone RNA polymerases. DNA viruses allow a larger genomic size and a richer gene repertoire to interact with the host and presumably evolve complex mechanisms for infectivity and survival; we have therefore restricted our study to DNA viruses. DNA viruses have utilized several mechanisms to evade host defenses [11]. Poxviruses and herpes viruses have evolved multiple strategies to disrupt presentation of viral antigens by MHC molecules in order to evade control by $\mathrm{T}$ lymphocytes [12]. The adenovirus inhibits MHC-I molecules to evade immune detection [13]. Viruses have also developed mechanisms to bolster the host cell to withstand the viral replication process such as an inhibition of apoptosis [14], an induction of cellular growth or differentiation [15], a recruitment of tissue repair processes such as fibrosis [16], tissue growth [17], and angiogenesis [18].

Evidence presented thus far suggests that viral miRNAs target host pathways and biological processes that may potentially benefit viral replication or persistence. If the suppression of a host gene disables host defense or enables viral survival or proliferation (e.g., by promoting cell growth, or inducing repair mechanisms to aid the survival of the infected cell), then encoding a miRNA that targets the specific host gene would potentially benefit the virus. While a few previous studies support this suggestion with specific examples in a limited context $[10,19,20]$, it is not clear whether there are shared patterns among different viral miRNAs targeting different hosts. Here we investigate the general patterns of cellular targeting by viral miRNAs for a comprehensive set of human and murine viruses.

We found that for several of the 135 known miRNAs encoded by human viruses, the putative human gene targets of these viral miRNAs are enriched for specific host pathways whose targeting is arguably beneficial to the virus. Many of these pathways, including cancer-related pathways, melanogenesis, axon guidance, ErbB, GnRH, Wnt and MAPK signaling pathways are independently 
enriched among targets of multiple miRNAs encoded by multiple viruses. Next, to assess the generality of our findings for known miRNAs, based on previously identified 6809 putative miRNAs encoded by 23 human viruses, we predicted high-confidence targets of the miRNAs. We then assessed the enrichment of pathways and Gene Ontology (GO) functional classes in the target sets. At a false discovery rate (FDR) of $10 \%$, we found that $75 \mathrm{miR}$ NAs, corresponding to 15 viruses, target 43 unique pathways. Of these pathways, 20 (6 of which are cancer-related) were repeatedly targeted by independent miRNAs encoded by 14 different viruses. A similar analysis of 4 murine viruses encoding 21 miRNAs also yielded several enriched pathways and functions which significantly overlapped with those identified in human. Based on our biological understanding, the most significant pathways and processes likely targeted by viral miRNAs present a reasonable choice from a virus' vantage point, which include cell growth, axon guidance, and antigen processing and presentation. We discuss this in light of current understanding of viral infection pathways. Moreover, based on genome sequences of 14 strains of human CMV, we found that the miRNAs targeting these specific pathways are evolutionarily more conserved than the other viral miRNAs, suggesting a purifying selection of targeting specific host pathways. Overall, our results suggest that viruses may have evolved their miRNA repertoire to target specific host pathways as a means for their survival and proliferation.

\section{Results}

Targets of experimentally validated Human viral miRNAs are enriched for several pathways and functions potentially important for viral infectivity and survival

There are 135 experimentally identified miRNAs for the human viruses included in our study, which are cataloged on miRBase (http://www.mirbase.org). For each of the 135 known miRNA, we predicted gene targets using the Miranda3.3 tool [21] in the 3' UTR sequences of 3504 human protein coding genes, which had unique ENTREZ IDs in KEGG [22]. We only considered targets with a Miranda score of at least 140 (which corresponds to a perfect heptamer match in positions 2-8). Beyond this baseline threshold, we scrambled each of the 3' UTR sequence and predicted targets in the scrambled sequences following an identical procedure and identified a score threshold at which $1 \%$ of the scrambled sequences had a target predicted. For the putative targets of each miRNA, we then identified KEGG pathways (http://www.kegg.jp) enriched in these target gene sets at $1 \%$ FDR (see Methods). Table 1 shows the pathways that were enriched in more than one virus, and in more than 3 independent miRNAs. As we discuss in detail later, many of these pathways - cancer, Axon guidance, ErbB, MAPK, and Wnt signaling, etc., are arguably beneficial targets for the virus.

\section{Pathways targeted by known miRNAs are also enriched among targets of predicted viral miRNAs}

Given that experimentally identified viral miRNAs represent a potentially very small fraction of all functional viral miRNAs there is merit to exploring high-confidence predicted viral miRNAs for evidence of targeting specific host pathways. We obtained predicted miRNA candidate hairpins for double stranded DNA (dsDNA) viruses from VirMir Database [23]. This includes 6809 miRNA for 23 human dsDNA viruses and 2188 miRNAs for 4 mouse dsDNA viruses (Table 2). We excluded the papillomaviridae family as it contained fewer than 10 predicted miRNAs. For each of the candidate miRNA, we then predicted gene targets as for the experimentally identified miRNAs described above. However, we used a miRNA-specific score threshold at which $5 \%$ of the scrambled sequences had a target predicted; this threshold was always much more stringent than the baseline threshold of 140 .

Next, as above, we tested whether the predicted viral miRNA targets are enriched for specific KEGG pathways. To accommodate for potentially high false positives in predicted miRNAs, we employed additional criteria for the selection of enriched pathways. After assessing the overlap between the predicted miRNA targets and the genes in the pathway using a hypergeometric test, we further adjusted

Table 1 Targets of known human viral miRNA are enriched for KEGG Pathways.

\begin{tabular}{|c|c|c|c|c|c|c|}
\hline KEGG Pathway Name & Total (135) & HSV1 (25) & HSV2 (24) & EBV (44) & CMV (17) & KSHV (25) \\
\hline Cancer & $10(27)$ & $2(3)$ & $2(4)$ & $2(3)$ & $1(6)$ & $3(11)$ \\
\hline ErbB signaling pathway & 9 & 3 & 2 & 4 & 0 & 0 \\
\hline Axon guidance & 7 & 3 & 1 & 1 & 0 & 2 \\
\hline Melanogenesis & 6 & 2 & 2 & 0 & 1 & 1 \\
\hline MAPK signaling pathway & 5 & 0 & 1 & 0 & 1 & 3 \\
\hline GnRH signaling pathway & 4 & 0 & 0 & 0 & 1 & 3 \\
\hline Wnt signaling pathway & 4 & 1 & 0 & 1 & 1 & 1 \\
\hline
\end{tabular}

Individual viral miRNAs target human genes that are enriched for KEGG pathways, and multiple viruses target the same pathways even across virus families. We list the pathways that were represented multiple times with a FDR of $1 \%$. The name of the virus and number of known miRNA used for analysis is supplied as the header; the values indicate the number of miRNA that are enriched for the pathway listed. Cancer pathways were aggregated into one category and the value indicates the number of miRNA that are enriched for a cancer-related pathway and in parenthesis is the total number of cancer pathways enriched. 
Table 2 Viral miRNA selection.

\begin{tabular}{|c|c|c|c|c|c|}
\hline Virus & miRNA & Virus & miRNA & Virus & miRNA \\
\hline Human adenovirus $A$ & 20 & Human adenovirus Type 35 & 54 & HSV6B & 107 \\
\hline Human adenovirus $\mathrm{C}$ & 104 & Human adenovirus Type 5 & 114 & HSV7 & 54 \\
\hline Human adenovirus D & 136 & Human adenovirus Type 7 & 78 & HHV8(KSHV) & 392 \\
\hline Human adenovirus $\mathrm{E}$ & 132 & HSV1 & 939 & Orf virus & 953 \\
\hline Human adenovirus $\mathrm{F}$ & 58 & HSV2 & 840 & Vaccinia virus & 38 \\
\hline Human adenovirus Type 11 & 52 & HSV3 (dumas) & 111 & Muridherpes 1 & 1158 \\
\hline Human adenovirus Type 1 & 122 & $\mathrm{HHV} 4(\mathrm{EBV})$ & 741 & Muridherpes 2 & 894 \\
\hline Human adenovirus Type 17 & 136 & $\mathrm{HHV} 5(\mathrm{CMV})$ & 1444 & Muridherpes 4 & 98 \\
\hline Human adenovirus Type 2 & 124 & HSV6 & 98 & Murine adenovirus $\mathrm{A}$ & 38 \\
\hline
\end{tabular}

Species specific viruses containing 10 or more miRNA were selected from VirMir DB. The virus name and number of miRNAa re listed.

p-values based on a randomized sampling approach in a pathway-specific fashion, controlling for the lengths of the target genes 3' UTR. Finally, we corrected the adjusted p-values for multiple testing (see Methods). Based on FDR threshold of $10 \%$, a total of 43 unique pathways were enriched for 75 miRNAs in 14 human viruses. Table 3 shows KEGG pathways that were enriched among the targets of miRNAs in more than two viruses.

We assessed whether the distribution of the number of miRNAs targeting a pathway is skewed relative to expectation. Given the bipartite graph composed of miRNAs (whose targets are enriched for specific pathways) and the corresponding enriched pathways, we randomized the bipartite graph while preserving the degree of each miRNA, in order to estimate the expected degree distribution of pathways. The degree distribution for the real data is skewed towards higher values (Kolmogorov Smirnov $(\mathrm{KS})$ test $\mathrm{p}$-value $=0.029)$. The antigen processing and presentation pathway was specifically enriched among the targets of twelve miRNA. Based on 1000 randomizations of the bipartite graph; the probability of this occurrence is less than 0.0001 . Three pathways were enriched in targets of 7 or more miRNAs ( $\mathrm{p}$-value = 0.016 based on 1000 randomizations).

\section{Human viral miRNA targets are enriched for specific GO functions}

While the pathway-enrichment analysis can reveal very specific biology, it is also limited by our knowledge of pathways. We therefore extended our pathway-enrichment procedure above to search for enriched GO biological processes. As before, we applied a term-specific adjustment of p-value, applied multiple testing corrections, and used a FDR threshold of $10 \%$ to select for enriched GO terms. Table 3 shows GO terms that were enriched among the targets of miRNAs in more than one virus. An interesting set of terms not revealed by KEGG pathway analysis became apparent (discussed later). As above, we analyzed

Table 3 Human miRNA targets are enriched for KEGG Pathways and GO terms, and multiple viruses target the same pathways even across virus families.

\begin{tabular}{|c|c|c|c|c|c|}
\hline \multirow[t]{2}{*}{ KEGG Pathway } & \multicolumn{2}{|c|}{ adeno/herpes/pox } & \multirow[t]{2}{*}{ Gene Ontology } & \multicolumn{2}{|c|}{ adeno/herpes/orf } \\
\hline & \#miRNA & \# virus & & \#miRNA & \# virus \\
\hline Biosynthesis of steroids & $2 / 1 / 0$ & $2 / 1 / 0$ & Cytoplasmic microtubule & $0 / 9 / 0$ & $0 / 3 / 0$ \\
\hline Glycerolipid metabolism & $0 / 3 / 1$ & $0 / 3 / 1$ & Microtubule binding & 0/9/0 & $0 / 2 / 0$ \\
\hline ErbB signaling pathway & $7 / 0 / 0$ & $4 / 0 / 0$ & Kinase binding & $0 / 8 / 0$ & $0 / 2 / 0$ \\
\hline Regulation of autophagy & $2 / 1 / 0$ & $2 / 1 / 0$ & Cytoplasmic vesicle membrane & $4 / 4 / 0$ & $4 / 3 / 0$ \\
\hline Hedgehog signaling pathway & $0 / 3 / 0$ & $0 / 2 / 0$ & Inner ear morphogenesis & 0/8/0 & $0 / 2 / 0$ \\
\hline Axon guidance & $3 / 5 / 0$ & $2 / 3 / 0$ & Multicellular organismal development & $0 / 7 / 0$ & $0 / 5 / 0$ \\
\hline Antigen processing and presentation & $3 / 6 / 3$ & $2 / 4 / 1$ & Transferrin transport & $4 / 3 / 0$ & $4 / 2 / 0$ \\
\hline Long-term potentiation & $0 / 4 / 0$ & $0 / 2 / 0$ & Protein autophosphorylation & $5 / 2 / 0$ & $5 / 1 / 0$ \\
\hline GnRH signaling pathway & $2 / 2 / 0$ & $2 / 2 / 0$ & Protein serine/threonine kinase activity & $0 / 5 / 1$ & $0 / 5 / 1$ \\
\hline \multirow[t]{5}{*}{ Cancer } & $3 / 17 / 7$ & $2 / 6 / 1$ & Extracell-glutamate-gated ion channel activity & $5 / 1 / 0$ & $4 / 1 / 0$ \\
\hline & & & Voltage-gated ion channel activity & $1 / 5 / 0$ & $1 / 3 / 0$ \\
\hline & & & Synaptic transmission & $1 / 5 / 0$ & $1 / 4 / 0$ \\
\hline & & & Histone methylation & $0 / 4 / 2$ & $0 / 3 / 1$ \\
\hline & & & Canonical Wnt Receptor signaling pathway & $0 / 4 / 2$ & $0 / 3 / 1$ \\
\hline
\end{tabular}

We list the pathways that were represented multiple times with an FDR of $10 \%$. The numbers separated by a slash correspond to adeno, herpes and orf families of viruses. 
the degree distribution associated with GO terms in the bipartite graph composed of enriched GO terms and miRNAs. As before, the GO terms were multiply targeted more frequently than expected (KS test p-value $=0.007$ ). Cytoplasmic microtubule and microtubule bindings were enriched for 9 miRNA.

It is possible that a pathway/GO term is enriched among targets of multiple miRNAs simply due to a high similarity of the miRNA sequences, and could not be interpreted as independent evidence. To rule out this possibility we analyzed the pair-wise overlap in target sites for miRNAa enriched the same functional term (see Methods). For each miRNA pair, we calculated the overlap as the fraction of all target sites (for the miRNA with fewer hits) that overlapped with the target sites of the other miRNA. We found that in almost all cases (99\%), the overlap was less than 0.05 . Thus, the miRNA sequence similarity does not explain the common pathway enrichment. Taken together, our results suggest that several pathways and GO terms are targeted independently by multiple miRNAs.

\section{Human and mouse viruses target similar processes}

Next, we investigated whether our finding in human - viral miRNAs tend to target critical host pathways - also holds in other species, and whether similar pathways and functions are targeted in evolutionarily distant species. We used herpes and adeno virus specific for mice and performed an identical KEGG pathway and GO term enrichment analyses as was used for human miRNAs. By necessity, the enrichment was restricted to genes with mapped unambiguous human-mouse orthologs; this reduced the size of the KEGG gene list for enrichment purposes from 3504 genes to 1381 genes, and the pathway list from 201 down to 189 . None of the miRNA target sets from the mouse adeno family were enriched for a functional term at the 10\% FDR threshold. For the herpes family, we found substantial overlap among the processes and pathways targeted by mouse and human viruses in their respective hosts, namely the ErbB signaling pathway, axon guidance, neuron development, regulation of immune response, and Insulin receptor regulation. Table 4 shows the pathways that were multiply targeted in at least one species. There was only one experimentally verified mouse miRNA included in our study and it was enriched for cell communication pathway, neuron differentiation, and cellular response to retinoic acid processes.

\section{Viral miRNAs targeting specific host processes are potentially evolving under purifying selection}

Many of the miRNAs encoded by EBV have been suggested to target host genes and were shown to be evolutionarily conserved [24]. Next, we investigated if the viral miRNAs that target specific host processes are evolving
Table 4 Mouse and human virus target similar KEGG pathways and GO terms.

\begin{tabular}{lc}
\hline KEGG Pathway & $\begin{array}{c}\text { Mouse/ } \\
\text { Human }\end{array}$ \\
\hline Synthesis and degradation of ketone bodies & $2 / 1$ \\
C21-steroid hormone metabolism & $1 / 2$ \\
ErbB signaling pathway & $2 / 4$ \\
Axon guidance & $2 / 3$ \\
Olfactory transduction & $2 / 2$ \\
Cancer & $2 / 5$ \\
\hline GO term & Mouse/ \\
& Human \\
\hline Multicellular organismal development & $3 / 5$ \\
Neuron development & $3 / 4$ \\
Regulation of immune response & $4 / 3$ \\
Peptidyl-tyrosine phosphorylation & $3 / 3$ \\
Cellular response to retinoic acid & $3 / 3$ \\
Integrin binding & $3 / 2$ \\
Intermediate filament & $3 / 2$ \\
Phosphorylation & $3 / 2$ \\
Positive regulation of muscle cell differentiation & $3 / 2$ \\
Negative regulation of insulin receptor signaling & $5 / 4$ \\
pathway & \\
Positive regulation of phosphatidylinositol 3-kinsase & $3 / 4$ \\
activity & $3 / 4$ \\
Insulin receptor signaling pathway & \\
\hline
\end{tabular}

Mouse and human viruses target genes sharing similar cellular signaling pathways and GO terms despite large divergence in 3'UTRs. KEGG pathways and GO terms which were enriched for multiple miRNA and multiple virus strains in one species are listed.

under differential pressure relative to other miRNAs. We obtained and aligned 14 CMV strain sequences [25] to reference the VirMir strain using ClustalW [26]. Given the multiple alignment we calculated the average conservation score for each miRNA, defined as a fraction of the consensus base at each position, averaged across the length of the miRNA. Those that putatively target specific host processes are significantly more conserved than the miRNAs that do not target a specific host pathway (Wilcoxon p-val 0.047), suggesting that they are evolving under purifying selection. This analysis could not be extended to other strains due to paucity of strains and inter-strain variability.

\section{Discussion}

Targeting specific host processes by viral miRNA are prevalent mechanisms of viral infectivity

Given that DNA viruses encode for miRNAs that can be effectively processed by the host machinery [27], it stands to reason that targeting of specific host genes by viral RNA may be effective ammunition against host defense. A single miRNA may target hundreds of genes for regulation, and identifying the true targets of miRNAs remains a challenge. This study was designed to investigate the 
possibility that virally-encoded miRNAs target host genes in pathways central to the persistence and/or replication strategies of many viruses. We first tested for enriched pathways among putative targets of known human viral miRNAs from miRbase. Recent next-generation sequencing efforts have revealed new viral miRNAs, and this trend is likely to continue as more cell types and tissues are analyzed [28] (and unpublished data by Trgovcich). Therefore, given that a vast majority of viral miRNAs remain to be identified, we used a dataset of predicted viral miRNA to test the generality of our findings for known miRNAs. Based on a stringent analysis, our results support the general hypothesis.

A few aspects of our finding are worth highlighting. First, published experimental data suggests that many of the pathways and processes putatively targeted by viral miRNAs make excellent targets to aid viral infectivity and survival. Second, we found that many KEGG pathways and GO biological processes are targeted by multiple miRNAs from multiple viruses, even across viral families. For instance, miRNAs targeting antigen processing and presentation and gonadotropin receptor hormone signaling are enriched in both adeno and herpes virus families. While it is known that herpes reside in neuronal cells during latency [29], axon guidance pathways are also enriched by both viral families, which is surprising because adeno viruses are normally associated with lung or gastrointestinal infections, and not neuronal cells [30]. The other commonality between both virus families was the cancerrelated pathways, which we categorized as one group. Third, we also found many of these enriched targets in human to be the same when we repeated our analysis independently in mouse viruses. For example, cancer and the ErbB signaling pathway, insulin receptor regulation, and axon guidance pathways are preserved between mouse and human viral targets. We discuss a selection of enriched pathway in more detail below. Finally, we found evidence to suggest that the miRNAs targeting specific host processes are evolving at a relatively slower rate than other miRNAs. This is consistent with previous findings in EBV that showed viral miRNAs are likely to target host genes that are evolutionarily conserved [24]. We note that the evolutionarily conserved miRNAs in our analysis are not biased towards known miRNAs.

A few previous studies report similar findings, although in a limited context. A previous analysis showed enrichment for KEGG pathways associated with cell signaling and adhesion/junction pathways using the putative targets of 85 known miRNAs in 5 human herpes virus as an aggregate (not virus-specific or miRNA-specific) [19]. Another study of known KSHV miRNAs and targets predicted by differential expression analysis found the targets to be involved in proliferation, immune modulation, angiogenesis, and apoptosis [31]. Finally experimentally identified targets for EBV were found to be enriched for targets involved in innate immunity, cell survival, and cell proliferation [10]. Here we verified these previous finding and extended the analysis to individual viruses and miRNAs as well as broadened the virus families to include the adenoviruses and poxviruses. Furthermore, by including human and mouse viruses, our analyses support evolutionary prevalence of viral miRNAs targeting host pathways and processes for their survival and proliferation. Finally, we present evidence for possible purifying selection, acting on certain miRNAs targeting specific host processes.

\section{Caveats}

While miRNA prediction is challenging in general, it is especially difficult to predict viral miRNAs, because most miRNA prediction approaches have been developed for eukaryotes and exploit evolutionary conservation [32], and are thus not applicable to viruses. Current miRNA prediction in viruses essentially relies on identifying hairpin like structures with certain additional properties [23]. We started with the putative miRNAs for dsDNA viruses compiled in the VirMir [23]. Undoubtedly, many of these predicted miRNAs will be false positives. However, the complete set of viral miRNAs is far from known. For instance, since 2007 in VirMir database, the number of known miRNAs for viruses studied here has grown greater than three-fold, and will grow as technologies improve and as additional cell types and tissues infected by viruses are analyzed. Using the group of all potential viral miRNAs based on predicted hairpin precursor structures therefore allowed us to begin with most comprehensive set of putative target genes for statistical analysis. Predicting miRNA targets presents yet another complication, especially for viruses, for similar reasons, and is fraught with false positives. A $24 \%$ false discovery rate has been calculated for MiRanda[33] similar to other target prediction algorithms. To minimize false positives from the putative miRNAs collected from VirMir, we restricted the target scores to a value of 140 or higher which correspond to exact seed matching criteria as well as a further threshold based on target score distribution for scrambled 3'UTR sequences. We adopted several additional checks to minimize false positives at the stage of functional enrichment assessment. First, we performed a strict pathway-specific adjustment of p-values accounting for 3' UTR lengths of genes in the pathway (a novel aspect of this work) and applied multiple testing corrections. Second, we used multiple virus families. Third, we relied on both KEGG and GO to better interpret our findings, and we used two distant species to corroborate our findings. Lastly, we compared the evolutionary conservation of miRNAs targeting specific host pathways to the other miRNAs based on 14 distinct strains of CMV and determined that they are conserved much more than 
expected, suggesting a selective pressure to maintain the targeting specificity. Although there are likely to be many false positives at the level of individual target genes, due to the reasons listed above, it is encouraging that they still reveal biologically meaningful patterns of functional enrichments.

\section{Pathways targeted by viral miRNAs}

We found that miRNAs in many viruses targeted similar pathways even across distant host species. We compared the pathway enrichment for known viral miRNA in miRBase with that for predicted miRNAs in VirMir. For brevity, we discuss in detail the biological significance of three most significant pathways.

\section{Cancer}

It is encouraging that cancer, as a theme, is repeatedly revealed by our analysis. It is becoming increasingly clear that miRNAs may have a causal role in initiation and progression of cancer [34]. A few viruses included in this study are known to be associated with human cancer such as EBV in Burkett's lymphoma and nasopharyngeal carcinoma, and KSHV in Kaposi's sarcoma and some B cell lymphomas, but there is no direct evidence yet that viral miRNAs play a causal role in the development of cancer. Oncogenic pathways that viruses are known to interfere with include (1) cell growth, (2) proliferation, (3) evasion of apoptosis, (4) genetic instability, and (5) angiogenesis. The enriched KEGG pathways by both mice and humans include one pathway and three GO terms associated with features deregulated in cancer, in addition to the cancer pathway. Regulation of the MAPK signaling pathway is an essential co-factor for re-activation of KSHV [35]. A closely related PI3K pathway, targeted by herpes viruses, is critical to cell survival and growth [36], and is frequently activated in cancer pathways [37]. Both pathways have the potential to inhibit KSHV infections [38]. The PI3K pathway is a particular favorite for DNA viruses enabling infected cells to withstand viral induced stress [39]. DNA viruses can affect ErbB signaling pathway, which can initiate the MAPK pathway resulting in growth or differentiation [40]. Enrichment for these pathways suggests that viral miRNAs may play a role in the oncogenesis.

\section{Hedgehog and insulin regulation}

The Hh genes regulate tissue patterning and are expressed in the beta cells of the pancreas and was implicated in insulin production [41]. Hepatitis infection induces Hh pathways and is associated with fibrogenic repair, and vascular remodeling [42]. The viral need for tissue repair could be categorized as stress management so that the infected cell can withstand the stresses of viral replication programs; however, it is not clear why a virus would need to regulate insulin. Insulin is a metabolic molecule that causes tissue to take up glucose from the blood and to stop using fat as an energy source; it is possibly used as a metabolic regulator. One can speculate that viral hijacking of the key energy regulator may induce the infected cell to devote their energy production to the virus' needs. Enrichment of the GO term for negative regulation of insulin receptor signaling and insulin receptor signaling pathway would indicate that insulin regulation is indeed the targeted pathway.

\section{Neuronal growth}

HSV is commonly latent in nerves, and during an infection the virus travels along the nerves to the connected tissue and forms lytic lesions. Nerve growth factors are necessary survival factors for HSV in latency [43]. Additionally, it has been shown that HSV utilizes nerve growth cones and varicosities of axons for transport [44] indicating a role in nerve growth pathways. What is surprising is that three miRNA from two different adeno viruses were enriched for axon guidance pathway in humans. Because adeno viruses do not usually target neurons, it is likely that this is a false positive. Nevertheless, viral miRNA targets are enriched for axon guidance and GO term neuron development and support the targeting of this important latency pathway for the herpes virus.

\section{Materials and methods MiRNA target prediction}

Given a miRNA sequence from VirMir [23], and the 3' UTR of human (or mouse) genes, we used Miranda3.3 to predict the miRNA targets [45]. As a baseline threshold, we only considered Miranda hits scoring 140 or higher, which corresponds to a perfect heptamer match in positions 2-8 and should be considered stringent [45]. As a further control, we randomly scrambled each 3' UTR sequence and repeated the target prediction on the randomized sequences. Using the distribution of scores on the randomized sequences, we determined a score cutoff corresponding to top $1 \%$ (for analysis of known miRNA) or $5 \%$ (for analysis of predicted miRNAs) scores. This threshold was determined separately for each miRNA and was always much more stringent that the baseline score threshold of 140 .

\section{Functional enrichment}

We compared each gene target set with an annotated functional gene sets corresponding to KEGG pathways and GO biological processes; gene sets with fewer than 10 members were excluded. For each miRNA we assessed overlap between the miRNA target set and each functional gene set using a hypergeometric test, which estimates the probability (p-value) of observing an overlap of certain size of greater between two gene sets by random chance. We noted that if the genes in functional gene set have longer 3' UTRs, then those pathways are more likely to be deemed as enriched simply by chance. To control 
for 3' UTR length bias, we divided the range of 3' UTR lengths into 20 equal-sized bins. For a miRNA, for each target of that miRNA, we randomly selected a gene in the same 20 percentile bin, thus generating a length matched random set of target genes for each miRNA; we generated 10 such random sets corresponding to each actual target set. For each randomize target set, we calculated the hypergeometric p-value for the overlap with each functional gene set. Thus, for a functional gene set (KEGG or GO), we generated a NULL distribution of hypergeometric $\mathrm{p}$-values containing $68,090 \mathrm{p}$-values corresponding to 10 randomized sets corresponding to 6809 miRNAs. Given an actual miRNA target set $S$ and functional gene set $F$, and given the hypergeometric $\mathrm{p}$-value $P(S, F)$, we estimated an adjusted p-value $P^{A d j}(\mathrm{~S}, \mathrm{~F})$ as the rank of $P$ in the 68,090 p-values corresponding to $F$. Finally, given adjusted p-values for all miRNA target sets for all viruses against all functional gene sets, we corrected the adjusted $\mathrm{p}$-values for multiple testing using the Benjamini-Hochberg procedure for FDR estimation [46].

\section{Additional files}

Predicted miRNA targets (310 unique Human miRNA, 817 unique Entrez gene ID's) are available at ftp://ftp. cbcb.umd.edu/pub/data/sridhar/Human.miRNA. supplemental.

\section{Authors' contributions}

S.H. and J.W.C. conceived and designed the project. J.W.C. performed the analyses. J.T. provided the virology expertise in selecting some of the datasets used as well as the discussion. S.H. and J.W.C. Wrote the manuscript with critical help from J.T.

\section{Competing interests}

The authors declare that there are no competing interests

\section{Acknowledgements}

This work is supported by $\mathrm{NIH}$ grant R01GM085226 to SH.

\section{Declarations}

The publication costs for this article were funded by $\mathrm{NIH}$ grant R01GM085226 to SH.

This article has been published as part of BMC Bioinformatics Volume 14 Supplement 2, 2013: Selected articles from the Eleventh Asia Pacific Bioinformatics Conference (APBC 2013): Bioinformatics. The full contents of the supplement are available online at http://www.biomedcentral.com/ bmcbioinformatics/supplements/14/S2.

\section{Author details}

${ }^{1}$ Center for Bioinformatics and Computational Biology, University of Maryland, College Park, MD, USA. ²Department of Surgery, The Ohio State University, Columbus, OH, USA.

Published: 21 January 2013

\section{References}

1. Ambros V: microRNAs: tiny regulators with great potential. Cell 2001, 107(7):823-826.
2. Neilson JR, Sharp PA: Herpesviruses throw a curve ball: new insights into microRNA biogenesis and evolution. Nat Methods 2005, 2(4):252-254.

3. Carrington JC, Ambros V: Role of microRNAs in plant and animal development. Science 2003, 301(5631):336-338.

4. Ambros V: The functions of animal microRNAs. Nature 2004, 431(7006):350-355.

5. Sayed DAM: MicroRNAs in development and disease. Physiol Rev 2011, 91(3):827-887.

6. Ding SWV: Antiviral immunity directed by small RNAs. Cell 2007, 130(3):413-426.

7. Otsuka MJQ, Georgel P, New L, Chen J, Mols J, Kang YJ, Jian Z, Du X, Cook R, Das SC, Pattnaik AK, Beutler B, Han J: Hypersusceptibility to vesicular stomatitis virus infection in Dicer1-deficient mice is due to impaired miR24 and miR93 expression. Immunity 2007, 27(1):123-134.

8. Umbach JLKM, Jurak I, Karnowski HW, Coen DM, Cullen BR: MicroRNAs expressed by herpes simiplex virus 1 during latent infection regulate viral mRNAs. Nature 2008, 454(7205):780-783.

9. Choy EYSK, Kok KH, Lung RW, Tsang CM, To KF, Kwong DL, Tsao SW, Jin DY: An Epstein-Barr virus encoded microRNA targets PUMA to promote host cell survival. J Exp Med 2008, 205(11):2551-2560.

10. Skalsky RL, Corcoran DL, Gottwein E, Frank CL, Kang D, Hafner M, Nusbaum JD, Feederle R, Delecluse H-J, Luftig MA, et al: The Viral and Cellular MicroRNA Targetome in Lymphoblastoid Cell Lines. PLoS Pathog 2012, 8(1):e1002484.

11. Alcami A, Koszinowski UH: Viral mechanisms of immune evasion. Immunology Today 2000, 21(9):447-455.

12. Horst DVM, Davison AJ, Ressing ME, Wiertz EJ: Viral evasion of T cell immunity: ancient mechanisms offering new applications. Current Opinion in Immunology 2011, 23(1):96-103.

13. Jiao JGH, Lippa AM, Ricciardi RP: The $\mathbf{N}$ terminus of adenovirus Type 12 E1A inhibits major histocompatibility complex class I expression by preventing phosphorylation of NF-kappaB p65 Ser276 through direct binding. Journal of virology 2010, 85(15):7668-7674.

14. Yee JWR, Anderton E, Allday MJ: Latent Epstein-Barr Virus Can Inhibit Apoptosis in B Cells by Blocking the Induction of NOXA Expression. PLoS One 2011, 6(12):e28506.

15. Blazara BAMA: Induction of $B$ cell responsiveness to growth factors by Epstein-Barr virus conversion: comparison of endogenous factors and interleukin-1. Clin Exp Immunol 1990, 80(1):62-68.

16. Kropski JALW, Blackwell TS: Right Place, Right Time: The evolving role of herpesvirus infection as a "second hit" in idiopathic pulmonary fibrosis. Am J Physiol Lung Cell Mol Physiol 2012, 302:L441-L444.

17. Byzova TVGC, Jankau J, Chen J, Cabrera G, Achen MG, Stacker Sa, Carnevale KA, Siemionow M, Deitcher SR, DiCorleto PE: Adenovirus encoding vascular endothelial growth factor-D induces tissues specific vascular patterns in vivo. Blood 2002, 99(12):4434-4442.

18. Sharma-Walia NPA, Bottero V, Sadagopan S, Veettil MV, Kerur N, Chandran B: Kaposi's sarcoma associated herpes virus (KSHV) induced COX-2: a key fator in latency, inflammation, angiogenesis, cell survival and invasion. PLoS Pathog 2010, 6(2):e1000777.

19. Gao GLJ, Kong L, Tao L, Wei L: Human herpesvirus miRNAs statistically perferentially target host genes involved in cell signaling and adhesion/ junction pathways. Cell Research 2009, 19(5):665-667.

20. Samols MASR, Maldonado AM, Riva A, Lopez MC, Baker HV, Renne R: Identification of cellular genes targeted by KSHV-encoded microRNAs. PLoS Pathog 2007, 3(5):e65.

21. Betel D, Koppal A, Agius P, Sander C, Leslie C: Comprehensive modeling of microRNA targets predicts functional non-conserved and non-canonical sites. Genome Biology 2010, 11(8):R90.

22. Kanehisa MGS: KEGG: kyoto encyclopedia of genes and genomes. Nucleic acids research 2000, 28(1):27-30.

23. Li SCSC, Lin WC: Vir-Mir db: prediction of viral microRNA candidate hairpins. Nucleic Acids Res 2008, 36:D184-D189.

24. Cai X, Schäfer A, Lu S, Bilello JP, Desrosiers RC, Edwards R, Raab-Traub N Cullen BR: Epstein-Barr Virus MicroRNAs Are Evolutionarily Conserved and Differentially Expressed. PLoS Pathog 2006, 2(3):e23.

25. Cunningham CGD, Hilfrich B, Baluchova K, Dargan DJ, Thomson M, Griffiths PD, Wilkinson GW, Schulz TF, Davison AJ: Sequences of complete human cytomegalovirus genomes from infected cell cultures and clinical specimens. The Journal of general virology 2010, 91(Pt 3):605-615. 
26. Thompson JDHD, Gibson TJ: CLUSTAL W: improving the sensitivity of progressive multiple sequence alignment through sequence weighting, position specific gap penalties and weight matrix choice. Nucleic Acids Res 1994, 22(22):4673-4680.

27. Pfeffer SZM, Grässer FA, Chien M, Russo JJ, Ju J, John B, Enright AJ, Marks D, Sander C, Tuschl T: Identification of virus-encoded microRNAs. Science 2004, 304(5671):734-736

28. Liu PFX, Feng Z, Guo YM, Peng RJ, Liu T, Huang Z, Feng Y, Sun X, Xiong Z, Guo X, Pang SS, Wang B, Lv X, Feng FT, Li DJ, Chen LZ, Feng QS, Huang WL, Zeng MS, Bei JX, Zhang Y, Zeng YX: Direct sequencing and characterization of a clinical isolate of Epstein-Barr virus from nasopharyngeal carcinoma tissue by using next-generation sequencing technology. Journal of virology 2011, 85(21):11291-11299.

29. Bloom DCSJ: Neuron-specific restriction of a herpes simplex virus recombinant maps to the UL5 gene. Journal of virology 1994, 68(6):3761-3772

30. Lynch JPFM, Echavarria M: Adenovirus. Seminars in respiratory and critical care medicine 2011, 32(4):494-511.

31. Gottwein ECD, Mukherjee N, Skalsky RL, Hafner M, Nusbaum JD, Shamulailatpam P, Love CL, Dave SS, Tuschl T, Ohler U, Cullen BR: Viral microRNA targetome of KSHV-infected primary effusion lymphoma cell lines. Cell Host Microbe 2011, 10(5):515-526.

32. Bartel DPCC: Micromanagers of gene expression: the potentially widespread influence of metazoan microRNAs. Nature reviews Genetics 2004, 5(5):396-400

33. Min HYS: Got target? Computational methods for microRNA target prediction and their extension. Experimental \& molecular medicine 2010 42(4):233-244.

34. Lovat FVN, Croce CM: MicroRNAs in the pathogenesis of cancer. Seminars in oncology 2011, 38(6):724-733.

35. Qin DFN, Fan W, Ma X, Yan Q, Lv Z, Zeng Y, Zhu J, Lu C: Activation of PI3K/AKT and ERK MAPK signal pathways is required for the induction of lytic cycle replication of Kaposi's sarcoma-associated herpesvirus by herpes simplex virus type 1. BMC microbiology 2011, 11.

36. Buchkovich NJYY, Zampieri CA, Alwine JC: The TORrid affairs of viruses: effects of mammalian DNA viruses on the PI3K-Akt-mTOR signalling pathway. Nature reviews Microbiology 2008, 6(4):266-275.

37. Fresno Vara JACE, de Castro J, Cejas P, Belda-Iniesta C, González-Barón M: PI3K/Akt signalling pathway and cancer. Cancer treatment reviews 2004, 30(2):193-204.

38. Lambert PJSA, Whitman AG, Dyson OF, Reber AJ, McCubrey JA, Akula SM: Targeting the PI3K and MAPK pathways to treat Kaposi's-sarcomaassociated herpes virus infection and pathogenesis. Expert opinion on therapeutic targets 2007, 11(5):589-599.

39. Buchkovich NJ, Maguire TG, Yu Y, Paton AW, Paton JC, Alwine JC: Human cytomegalovirus specifically controls the levels of the endoplasmic reticulum chaperone BiP/GRP78, which is required for virion assembly. J Virol 2008, 82(1):31-39.

40. Tzahar EMJ, Waterman H, Barbacci EG, Bao J, Levkowitz G, Shelly M, Strano S, Pinkas-Kramarski R, Pierce JH, Andrews GC, Yarden Y: Pathogenic poxviruses reveal viral strategies to exploit the ErbB signaling network. The EMBO journal 1998, 17(20):5948-5963.

41. Thomas MKRN, Lee JH, Habener JF: Hedgehog signaling regulation of insulin production by pancreatic beta-cells. Diabetes 2000, 49(12):2039-2047.

42. Pereira Tde AWR, Syn WK, Choi SS, Bradrick S, Karaca GF, Agboola KM Jung Y, Omenetti A, Moylan CA, Yang L, Fernandez-Zapico ME, Jhaveri R, Shah VH, Pereira FE, Diehl AM: Viral factors induce Hedgehog pathway activation in humans with viral hepatitis, cirrhosis, and hepatocellular carcinoma. Laboratory investigation; a journal of technical methods and pathology 2010, 90(12):1690-1703.

43. Wilcox CLSR, Freed CR, Johnson EM Jr: Nerve growth factor-dependence of herpes simplex virus latency in peripheral sympathetic and sensory neurons in vitro. J Neurosci 1990, 10(4):1268-1275.

44. Saksena MMWH, Tijono B, Boadle RA, Rixon F, Takahashi H, Cunningham AL: Herpes simplex virus type 1 accumulation, envelopment, and exit in growth cones and varicosities in mid-distal regions of axons. Journal of virology 2006, 80(7):3592-3606.

45. Betel DKA, Agius P, Sander C, Leslie C: Comprehensive modeling of microRNA targets predicts functional non-conserved and non-canonical sites. Genome Biol 2010, 11(8):R90.
46. Benjamini Y, Hochberg Y: Controlling the False Discovery Rate: A Practical and Powerful Approach to Multiple Testing. Journal of the Royal Statistical Society Series B (Methodological) 1995, 57(1):289-300.

doi:10.1186/1471-2105-14-S2-S3

Cite this article as: Carl et al.: Widespread evidence of viral miRNAs targeting host pathways. BMC Bioinformatics 2013 14(Suppl 2):S3.

\section{Submit your next manuscript to BioMed Central and take full advantage of:}

- Convenient online submission

- Thorough peer review

- No space constraints or color figure charges

- Immediate publication on acceptance

- Inclusion in PubMed, CAS, Scopus and Google Scholar

- Research which is freely available for redistribution 